\title{
Influence of conducting plate boundary conditions on the transverse envelope equations describing intense ion beam transport
}

\author{
Steven M. Lund* \\ Lawrence Livermore National Laboratory, University of California, Livermore, California 94550, USA \\ Boris Bukh \\ Lawrence Berkeley National Laboratory, University of California, Berkeley, California 94720, USA
}

(Received 22 July 2003; revised manuscript received 23 February 2004; published 4 June 2004)

\begin{abstract}
In typical diagnostic applications, intense ion beams are intercepted by a conducting plate associated with devices used to measure beam phase-space projections. This results in the transverse space-charge field near the plate being shorted out, rendering simple envelope models with constant space-charge strength inaccurate. Here we develop corrected envelope models based on analytical calculations to account for this effect on the space-charge term of the envelope equations, thereby removing a systematic source of error in the equations and enabling more accurate comparisons with experiment. For common intense beam parameters, we find that the envelope correction occurs primarily in the envelope angles near the plate and that the effect can be large enough to degrade precision beam matching in periodic transport lattices. Results are verified with 3D self-consistent particle-in-cell simulations based on intense beam experiments associated with driver development for heavy-ion fusion.
\end{abstract}

DOI: 10.1103/PhysRevSTAB.7.064201

PACS numbers: 29.27.Bd, 41.75.-i, 52.59.Sa, 52.27.Jt

\section{INTRODUCTION}

Reduced models of intense ion beams often employ the rms envelope equations to simply describe the self-consistent evolution of the statistical beam edge in response to applied focusing, space-charge, and thermal defocusing forces [1-4]. Such envelope models are typically solved with constant rms beam emittances (statistical measures of beam phase-space area) and constant perveance (space-charge strength) to extrapolate experimental measurements and understand the evolution of the beam envelope away from diagnostic stations. More complete descriptions of beam evolution employ the Vlasov model and must typically be solved by numerical simulations that can be difficult and computationally intensive. Therefore, developing easy to solve and reliable reduced envelope descriptions of the beam carries practical value.

A typical slit-scanner intercepting beam diagnostic used to measure transverse beam phase-space projections is sketched in Fig. 1. In this diagnostic an elliptical crosssection beam emerging from a transport channel free drifts into a conducting plate with a thin slit that passes a thin ribbon of particles (sized for adequate signal while maintaining good resolution) that is then intercepted by a second nearby slit plate. The second thin slit is parallel to the first slit and is combined with a Faraday cup to collect the transmitted component of the beam distribution.

*Electronic address: smlund@lbl.gov
By differentially moving the plates in directions perpendicular to the slit axes and recording signals collected, phase-space projections of the beam distribution perpendicular to the slit axis can be unfolded at the axial location of the first plate $[5,6]$. Sequences of such diagnostics with orthogonal slits are often employed to measure the evolution of transverse beam phase-space projections from which beam envelope parameters are calculated. Alternatively, optical beam imagers have been employed to measure more complete phase-space data of the beam distribution and envelope projections are made [7]. Both classes of intercepting beam diagnostics are characterized in an approximate fashion by a conducting plate that intercepts the beam with near normal incidence at the axial plane of the measurement. The plate will alter the electrostatic self-field produced by the space charge of an intense beam which will in turn alter the dynamics of particles and the transverse beam envelope near the plate relative to the usual situation where the plate is not present when no measurements are made. Developing a simple model to compensate for systematic changes in the transverse envelope induced by such plates is needed for more precise estimates of the envelope of beam particles without the need for large simulations. Elimination of systematic errors in envelope modeling can improve the precision of envelope matching which is important in limiting the generation of beam halo and related particle losses.

This paper is organized as follows. In Sec. II an electrostatic beam envelope model is derived with form factor corrections to the usual space-charge terms in the 


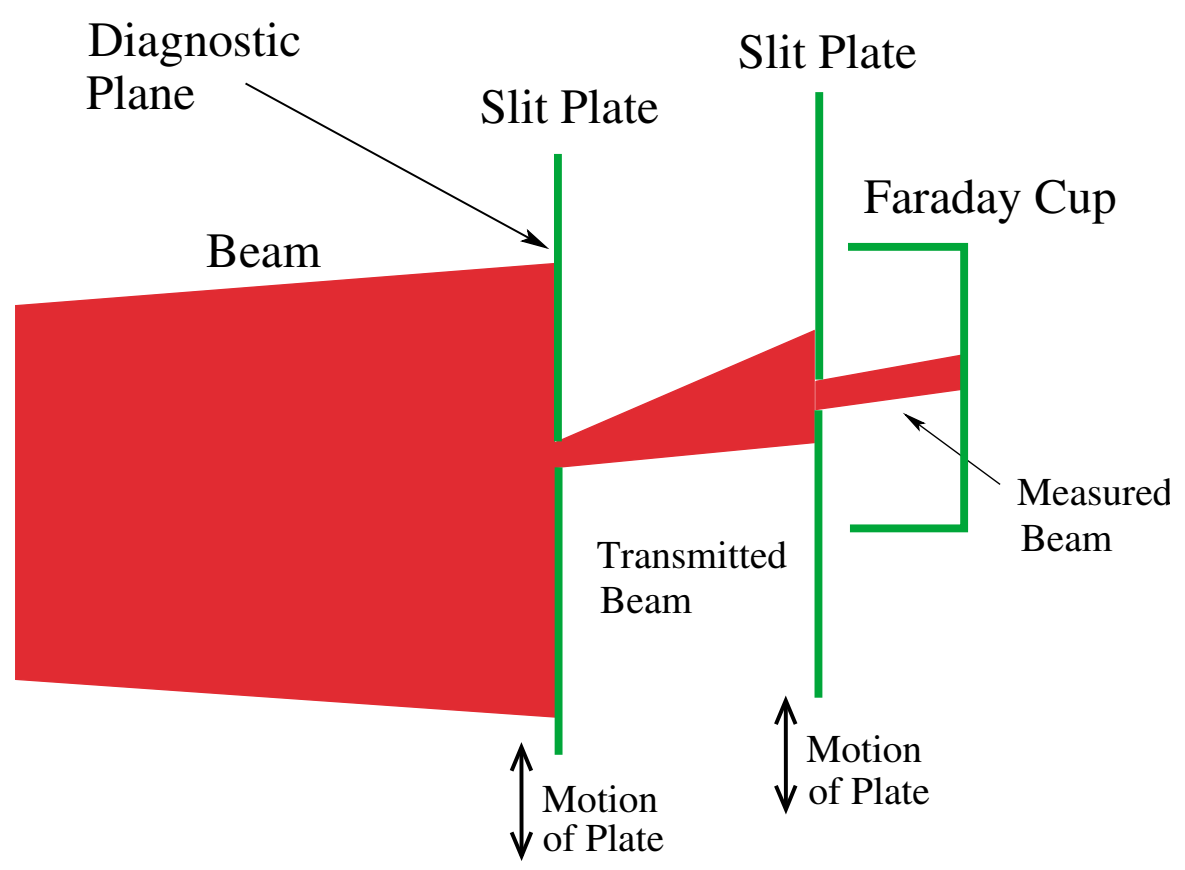

FIG. 1. (Color) Cross section of slit-plate diagnostics for measurement of beam phase space.

envelope equations. These form factors can be used to calculate corrections from a variety of self-field effects and are used in this study to evaluate the effect of an intercepting conducting plate altering beam space-charge fields. A simple plane in free-space model of the plate is adopted and an image-charge solution for the beam selffields is used to derive explicit expressions for the form factor corrections in a form convenient for analytical modeling. Particle-in-cell (PIC) simulations of a more realistic version of this geometry based on practical experiments are described in Sec. III. These simulations are used to check assumptions made in Secs. IV and V to simplify envelope models. The space-charge model is solved for a uniform-density, axisymmetric $(\partial / \partial \theta=0)$ beam in Sec. IV. Analytical field solutions are employed to calculate form factors and derive a heuristic corrected envelope equation for an axisymmetric beam in the presence of the conducting plate. Model predictions are verified with PIC simulations. Insights gained in Sec. IV are then applied to the more difficult case of a uniformdensity elliptical beam in Sec. V, where a more approximate corrected envelope equation is derived and again verified with simulations.

\section{ENVELOPE MODEL}

Consider a long-pulse, unbunched beam with particles of charge $q$ and mass $m$ moving with axial velocity $\beta_{b} c$ ( $c$ is the speed of light in vасииo) and relativistic factor $\gamma_{b}=1 / \sqrt{1-\beta_{b}^{2}}$. We take the transverse orbits $x(s)$ and $y(s)$ of a beam particle to satisfy the paraxial (radial energy variation of particles neglected) equations of motion [1]

$$
\begin{aligned}
& x^{\prime \prime}+\frac{\left(\gamma_{b} \beta_{b}\right)^{\prime}}{\left(\gamma_{b} \beta_{b}\right)} x^{\prime}+\kappa_{x} x+\frac{q}{m \gamma_{b}^{3} \beta_{b}^{2} c^{2}} \frac{\partial \phi}{\partial x}=0, \\
& y^{\prime \prime}+\frac{\left(\gamma_{b} \beta_{b}\right)^{\prime}}{\left(\gamma_{b} \beta_{b}\right)} y^{\prime}+\kappa_{y} y+\frac{q}{m \gamma_{b}^{3} \beta_{b}^{2} c^{2}} \frac{\partial \phi}{\partial y}=0 .
\end{aligned}
$$

Here, $s$ is the axial coordinate of a beam slice, primes denote derivatives with respect to $s, \kappa_{x}(s)$ and $\kappa_{y}(s)$ are the linear applied focusing functions of the lattice, and the electrostatic potential $\phi$ is related to the number density of beam particles $n$ by the 3D Poisson equation

$$
\nabla^{2} \phi=-\frac{q}{\epsilon_{0}} n
$$

subject to $\phi=$ const on conducting boundaries. Here, $\epsilon_{0}$ is the permittivity of free space, and SI units are employed except where otherwise noted. Specific forms of the focusing functions $\kappa_{x}$ and $\kappa_{y}$ are given for various classes of transport lattices in Ref. [2]. The terms $\left(\gamma_{b} \beta_{b}\right)^{\prime} x^{\prime} /\left(\gamma_{b} \beta_{b}\right)$ and $\left(\gamma_{b} \beta_{b}\right)^{\prime} y^{\prime} /\left(\gamma_{b} \beta_{b}\right)$ in Eq. (1) are associated with any acceleration of the beam slice which changes $\beta_{b}$ and $\gamma_{b}$. Such acceleration effects must be calculated consistently with longitudinal particle dynamics and can also result in changes in $\kappa_{x}$ and $\kappa_{y}$ depending on the particular applied focusing system employed.

Denote a transverse statistical average over an axial slice of beam particles by $\langle\cdots\rangle_{\perp}$. rms measures of the transverse edge radii of the beam envelope are

$$
r_{x}(s)=2 \sqrt{\left\langle x^{2}\right\rangle_{\perp}}, \quad r_{y}(s)=2 \sqrt{\left\langle y^{2}\right\rangle_{\perp}}
$$

The statistical envelope radii $r_{x}$ and $r_{y}$ correspond to the transverse edge radii of a uniform-density beam slice of 
elliptical transverse cross section with principal axes aligned with the $x$ - and $y$-coordinate axes and centered at $x=0=y$ (i.e., $\langle x\rangle_{\perp}=0=\langle y\rangle_{\perp}$ ). Differentiating the equations for $r_{x}$ and $r_{y}$ and employing Eq. (1) yields the envelope equations

$$
\begin{aligned}
& r_{x}^{\prime \prime}+\frac{\left(\gamma_{b} \beta_{b}\right)^{\prime}}{\left(\gamma_{b} \beta_{b}\right)} r_{x}+\kappa_{x} r_{x}-\frac{2 Q}{r_{x}+r_{y}} F_{x}-\frac{\varepsilon_{x}^{2}}{r_{x}^{3}}=0, \\
& r_{y}^{\prime \prime}+\frac{\left(\gamma_{b} \beta_{b}\right)^{\prime}}{\left(\gamma_{b} \beta_{b}\right)} r_{y}+\kappa_{y} r_{y}-\frac{2 Q}{r_{x}+r_{y}} F_{y}-\frac{\varepsilon_{y}^{2}}{r_{y}^{3}}=0 .
\end{aligned}
$$

Here,

$$
Q=\frac{q \lambda}{2 \pi \epsilon_{0} m c^{2} \gamma_{b}^{3} \beta_{b}^{2}}=\text { const }
$$

is the dimensionless perveance $\left(\lambda=q \int d x d y n=\right.$ const is the line-charge density of the beam slice),

$$
\begin{aligned}
& F_{x}=-\frac{4 \pi \epsilon_{0}}{\lambda}\left(\frac{r_{x}+r_{y}}{r_{x}}\right)\left\langle x \frac{\partial \phi}{\partial x}\right\rangle_{\perp}, \\
& F_{y}=-\frac{4 \pi \epsilon_{0}}{\lambda}\left(\frac{r_{x}+r_{y}}{r_{y}}\right)\left\langle y \frac{\partial \phi}{\partial y}\right\rangle_{\perp}
\end{aligned}
$$

are form factors, and

$$
\begin{aligned}
& \varepsilon_{x}=4\left[\left\langle x^{2}\right\rangle_{\perp}\left\langle x^{\prime 2}\right\rangle_{\perp}-\left\langle x x^{\prime}\right\rangle_{\perp}^{2}\right]^{1 / 2}, \\
& \varepsilon_{y}=4\left[\left\langle y^{2}\right\rangle_{\perp}\left\langle y^{\prime 2}\right\rangle_{\perp}-\left\langle y y^{\prime}\right\rangle_{\perp}^{2}\right]^{1 / 2}
\end{aligned}
$$

are the rms edge emittances which correspond to statistical measures of beam phase-space area in $x-x^{\prime}$ and $y-y^{\prime}[1]$.

For the special case of a $2 \mathrm{D}(\partial / \partial z=0)$ transverse beam in free space with elliptical symmetry charge density [i.e., $n=n(\rho)$ with $\rho=\sqrt{x^{2} / r_{x}^{2}+y^{2} / r_{y}^{2}}$, Sacherer [3] analyzed beam self-fields and showed that $F_{x}=F_{y}=1$. The Vlasov model self-consistent Kapchinskij-Vladimirskij distribution satisfies this condition for the special case of a uniform-density elliptical beam with constant normalized emittances $\varepsilon_{n x} \equiv$ $\gamma_{b} \beta_{b} \varepsilon_{x}=$ const and $\varepsilon_{n y} \equiv \gamma_{b} \beta_{b} \varepsilon_{y}=$ const $[1,4]$. The envelope equations (4) are also often applied with $F_{x}=$ $F_{y}=1$ to nonuniform density beams with evolving normalized emittances in an rms equivalent beam sense $[1,3]$. By calculating the form factors $F_{x}$ and $F_{y}$ as functions of $r_{x}$ and $r_{y}$ (and possibly other $s$-varying quantities) in specific geometries for a given (possibly selfconsistently evolving) beam charge distribution, the envelope equations (4) can be compensated for effects such as evolving space-charge nonuniformities and conductor boundary conditions (often called image charges). In this paper we address a specific form of image-charge compensations associated with conducting plates intercepting the beam. Formally, Eqs. (4) are consistent with constant normalized emittances only when the electric self-field components

$$
E_{x}=-\frac{\partial \phi}{\partial x}, \quad E_{y}=-\frac{\partial \phi}{\partial y}
$$

used in calculating $F_{x}$ and $F_{y}$ are linear functions of $x$ and $y$, respectively, within the beam. However, Eqs. (4) are sometimes solved with $F_{x}$ and $F_{y}$ calculated with nonlinear terms in $E_{x}$ and $E_{y}$ (i.e., $E_{x}$ containing terms $\propto x^{2}$, $\left.x y, x^{3}, \ldots\right)$ and assumed constant normalized emittances. The efficacy of such nonconsistent orderings must be established for logical consistency if nonlinear field terms are employed to claim more accurate estimates of envelope evolutions with Eqs.;(4) because emittance evolutions consistent with self-field nonlinearities can also influence the envelope evolution. Unfortunately, such consistency checks have rarely been carried out in the literature when nonlinear self-field terms are included in form factor corrections to the envelope equations. In Sec. IV we address this issue by making comparisons of corrected moment envelope model results derived with both linear and nonlinear self-field models to self-consistent PIC simulations.

For purposes of deriving analytical models, we idealize the geometry as a beam impinging on a perfectly conducting plane at $z=0$ in free space from $z<0$ as sketched in Fig. 2. In this situation the method of images can be used to solve for $\phi$ in the beam region with $z<0$ as

$$
\phi(\mathbf{x})=\frac{q}{4 \pi \epsilon_{0}} \int_{\text {beam }} d^{3} \tilde{x}\left[\frac{n(\tilde{\mathbf{x}})}{|\mathbf{x}-\tilde{\mathbf{x}}|}-\frac{n\left(\tilde{\mathbf{x}}_{I}\right)}{\left|\mathbf{x}-\tilde{\mathbf{x}}_{I}\right|}\right]
$$

Here $\mathbf{x}=x \hat{\mathbf{x}}+y \hat{\mathbf{y}}+z \hat{\mathbf{z}}$ and $\mathbf{x}_{I}=x \hat{\mathbf{x}}+y \hat{\mathbf{y}}-z \hat{\mathbf{z}}$ are the direct and image coordinates of a beam particle, and we have dropped an arbitrary additive constant to $\phi$ consistent with taking a bias $\phi=0$ on the plate. For transverse effects the value of the plate bias is not important if the energy of the beam is sufficiently high (specified more concretely later). However, if longitudinal acceleration effects induced by the plate are also evaluated, the choice of plate bias can become important.

\section{PARTICLE-IN-CELL SIMULATIONS}

Self-consistent 3D electrostatic PIC simulations are carried out to validate approximations and model assumptions that are made in subsequent sections to derive approximate form factors and enable direct solution of the envelope model. The simulations allow analysis of model deviations resulting from more realistic geometry, self-field nonlinearities, emittance growth, rapid variations in the beam envelope near the plate, energy deviations (acceleration) due to the beam seeing its image in the plate, and effects resulting from deviations in the beam transverse cross sections from simple uniformdensity elliptical. In this section we describe the general features of the simulations and numerical parameters. 
Simulation results are given in Secs. IV and V where comparisons to reduced analytical models are made.

Simulation parameters are based on typical diagnostic measurements in the High Current Experiment (HCX) for heavy-ion fusion (HIF) $[5,6,8]$, where a coasting $\left(\beta_{b}=\right.$ const) intense $K^{+}$ion beam with particle kinetic energy $\mathcal{E}_{b}=\left(\gamma_{b}-1\right) m c^{2}=1.0-1.7 \mathrm{MeV}$ is focused in a FODO quadrupole lattice [2] with period $L_{p}=435.2 \mathrm{~mm}$. At $\mathcal{E}_{b}=1.0 \mathrm{MeV}$, injected beam currents are in the range $I \simeq \lambda / \sqrt{2 \mathcal{E}_{b} / \mathrm{m}}=180-250 \mathrm{~mA}$ (corresponding to $Q \sim$ $\left.7-10 \times 10^{-4}\right)$ with beam emittances $\varepsilon_{x} \sim \varepsilon_{y} \sim$ 50-100 $\mathrm{mm}$ mrad. Electric quadrupole focusing strengths are tuned for an undepressed single-particle phase advance of $\sigma_{0}=40^{\circ}-90^{\circ}$ per lattice period. For quadrupole focusing strength with $\sigma_{0}=80^{\circ}$, a typical matched beam envelope in the HCX periodic transport channel has average envelope radius $\left(1 / L_{p}\right) \int_{0}^{L_{p}} d s r_{x}(s) \sim 10 \mathrm{~mm}$ and maximum envelope angles are $\max \left[\left|r_{x}^{\prime}\right|\right] \sim 50 \mathrm{mrad}$. Free drifts $\left(\kappa_{x}=\kappa_{y}=0\right)$ from the exit of quadrupole focusing elements to conducting plates of slit-scanner diagnostic stations are $\sim 60-120 \mathrm{~mm}$.

We employ the 3D WARP code developed for simulation of intense beams in HIF applications [9]. This code has an extensive hierarchy of models allowing both checks of numerical methods and idealizations made. A multigrid field solver is employed that allows boundaries of detailed conductor structures to be placed at subgrid resolution on the regular parallelepiped grid of the code. To represent HCX-like beams, we carry out steady-state, midpulse simulations of a beam injected into a focus-free drift section. In all simulations presented we take $\mathcal{E}_{b}=$ $1.0 \mathrm{MeV}$, singly ionized ions with mass $m=39.1 \mathrm{amu}$, and the injected beam current is varied to attain a specified perveance $Q$. The drift is $70 \mathrm{~mm}$ long axially. To reduce the idealization of the geometry taken in Fig. 2, a grounded $(\phi=0)$ cylindrical conducting pipe with radius $r_{p}=100 \mathrm{~mm}$ is added. Such pipes or other structures that reduce longitudinal self-field components of the beam are often present in experiments. The beam is injected from the left side of the grid $(s=0)$ with $\partial \phi / \partial z=0$ to model a beam entering from a long focusing channel. On the right side of the grid, the conducting plate at the diagnostic plane $\left(s=s_{p}=70 \mathrm{~mm}\right)$ is held at $\phi=0$. The injected beam is "semi-Gaussian" with a uniform distribution of particle coordinates $x$ and $y$ within an elliptical beam envelope with principal radii $r_{x}$ and $r_{y}$ along the transverse $x$ and $y$ axes. The injected semi-Gaussian beam also has particle angles $x^{\prime}$ and $y^{\prime}$ with coherent components $r_{x}^{\prime}\left(x / r_{x}\right)$ and $r_{y}^{\prime}\left(y / r_{y}\right)$ and incoherent spatially uniform, Gaussian distributed spreads in angles with variances set such that the specified emittances $\varepsilon_{x}$ and $\varepsilon_{y}$ are injected. This injection condition is a reasonable approximation to a relaxed, strongly spacecharge dominated beam emerging from a long transport channel where the density is expected to be nearly uniform and the beam edge sharp [10]. The injected incoherent longitudinal velocity spread of the beam is Gaussian distributed with variance set such that the spread in longitudinal particle velocities about the mean velocity set by the specified particle kinetic energy is equal to half

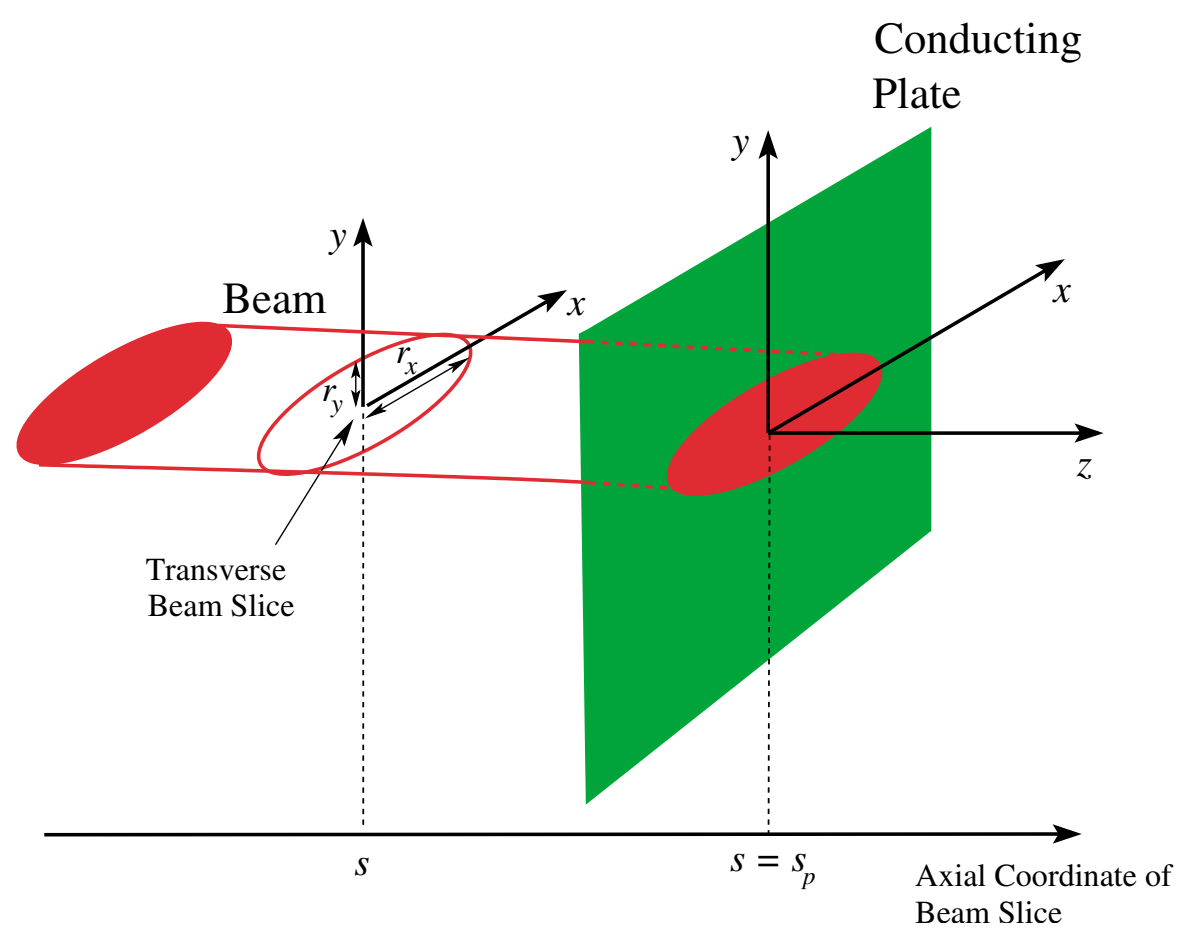

FIG. 2. (Color) Geometry of an unbunched beam incident on a conducting plane from the left $(z<0)$. 
the transverse spread in incoherent particle velocities (i.e., the longitudinal temperature in the beam frame is half the transverse temperature). No coherent spread in axial velocity is taken across the transverse profile of the injected beam. For the strong space-charge parameters considered in this study, we saw little difference from the injection condition taken compared to simulations done with a relaxed matched beam in a long periodic (or continuous) focusing channel that terminates with a drift near the diagnostic plate. A beam emerging from a long focusing channel may have a small coherent spread in axial velocity across the profile of the beam. Characteristics of this spread will depend on the beam injector and the details of the transport.

Numerical parameters of the simulations are set for high resolution to resolve nonlinear space-charge fields and a sharp beam edge. Spatial grids are uniform with typical transverse grid increments $d x=d y \sim$ $0.2-0.4 \mathrm{~mm}$ and axial grid increment $d z \sim 0.2-0.8 \mathrm{~mm}$, corresponding to $\sim 25-50$ grids across the transverse radius of the beam and $\sim 80-350$ grids along the longitudinal axis of the beam. An axisymmetric $r-z$ field solver is used in place of the full three-dimensional field solver in cases where an axisymmetric beam is injected with $r_{x}(0)=r_{y}(0)$ and $r_{x}^{\prime}(0)=r_{y}^{\prime}(0)$, and 4-fold transverse symmetry is used for elliptical beam injections with $r_{x}(0) \neq r_{y}(0)$ and/or $r_{x}^{\prime}(0) \neq r_{y}^{\prime}(0)$. Exploiting these symmetries allows more rapid simulations and improved statistics. The same three-dimensional particle mover is used for both axisymmetric and nonaxisymmetric injections. Particles are advanced in time with periodic field solves (subcycled relative to particle advances to reduce simulation time, with 5-20 advances per field solve) from injection until exiting the grid at the diagnostic plate where the particle disappears in the simulation. Particles are typically injected for two transit times through the axial grid, allowing transients to propagate off the grid to attain a steady midpulse solution. More than $7 \times 10^{6}$ macroparticles with smoothed interactions are used to represent the beam filling the grid on the steady-state solution to reduce statistical noise in the calculation of self-fields and better represent the ideal Vlasov evolution. All simulation results are converged to the accuracy presented with respect to time step, gridding, and particle statistics.

\section{CORRECTED ENVELOPE EQUATIONS FOR AXISYMMETRIC BEAMS}

Before proceeding to analyze the more difficult case of an elliptical beam in Sec. V, we first develop modeling techniques for an axisymmetric $(\partial / \partial \theta=0)$ beam with $r_{x}(s)=r_{y}(s)=R(s)[11]$.

\section{A. Self-field solution}

We further idealize the beam self-field solution given by Eq. (9) by assuming that the beam is normally incident with uniform density and a constant, round edge radius ( $r_{x}=r_{y}=R=$ const). Then the beam density is given by

$$
n(r, z)=\frac{\lambda}{\pi q R^{2}} \Theta(R-r) \Theta(-z),
$$

where $\Theta(x)$ is the Heaviside step function $[\Theta(x)=0$ for $x<0$ and $\Theta(x)=1$ for $x>0]$. In $(r, \theta, z)$ cylindrical coordinates with $x=r \cos \theta$ and $y=r \sin \theta, 1 /|\mathbf{x}-\tilde{\mathbf{x}}|$ can be expanded as [12]

$$
\frac{1}{|\mathbf{x}-\tilde{\mathbf{x}}|}=\sum_{\nu=-\infty}^{\infty} \int_{0}^{\infty} d k e^{i \nu(\theta-\tilde{\theta})} J_{\nu}(k r) J_{\nu}(k \tilde{r}) e^{k\left(z_{>}-z_{<}\right)},
$$

where $z_{>}$and $z_{<}$denote the greater and lesser of $z$ and $\tilde{z}$, and $J_{\nu}(x)$ is the $\nu$ th-order ordinary Bessel function. Using this expansion and Eq. (10) in Eq. (9) gives for $z<0$

$$
\phi(r, z)=\frac{\lambda}{\pi \epsilon_{0}} \int_{0}^{\infty} \frac{d w}{w^{2}}\left(1-e^{-w|z| / R}\right) J_{0}\left(w \frac{r}{R}\right) J_{1}(w),
$$

and the corresponding radial and axial electric field components $E_{r}=-\partial \phi / \partial r$ and $E_{z}=-\partial \phi / \partial z$ are

$$
\begin{aligned}
& E_{r}(r, z)=\frac{\lambda}{\pi \epsilon_{0} R} \int_{0}^{\infty} \frac{d w}{w}\left(1-e^{-w|z| / R}\right) J_{1}\left(w \frac{r}{R}\right) J_{1}(w), \\
& E_{z}(r, z)=\frac{\lambda}{\pi \epsilon_{0} R} \int_{0}^{\infty} \frac{d w}{w} e^{-w|z| / R} J_{0}\left(w \frac{r}{R}\right) J_{1}(w) .
\end{aligned}
$$

These field components are plotted in Fig. 3. Note that the radial field remains nearly linear within the beam $(r<R)$ until $z$ is a fraction of a beam radius from the plate. The axial field increases with decreasing $|z|$ because the negative image beam becomes closer as the plate is approached.

Equations (12) can be partially checked by calculating the radial field far from the plate and the longitudinal field on axis $(r=0)$ :

$$
\begin{aligned}
\lim _{|z| \rightarrow \infty} E_{r}(r, z) & =\frac{\lambda}{\pi \epsilon_{0} R} \int_{0}^{\infty} \frac{d w}{w} J_{1}\left(w \frac{r}{R}\right) J_{1}(w) \\
& =\frac{\lambda}{2 \pi \epsilon_{0} R} \begin{cases}\frac{r}{R}, & 0 \leq \frac{r}{R} \leq 1 \\
\frac{1}{r / R}, & 1 \leq \frac{r}{R}\end{cases} \\
E_{z}(r=0, z) & =\frac{\lambda}{\pi \epsilon_{0} R} \int_{0}^{\infty} \frac{d w}{w} e^{-w|z| / R} J_{1}(w) \\
& =\frac{\lambda}{\pi \epsilon_{0} R^{2}}\left(\sqrt{R^{2}+z^{2}}-|z|\right) .
\end{aligned}
$$

The radial field limit is the usual expression for a uniform-density beam of radius $R$. The expression for the on-axis field $E_{z}(r=0, z)$ shows that $\phi(r=0, z)$ logarithmically diverges in $|z|$ with

$$
\begin{aligned}
\phi(r=0, z)=\frac{\lambda}{2 \pi \epsilon_{0} R^{2}}( & |z| \sqrt{R^{2}+z^{2}}-z^{2} \\
& \left.+R^{2} \ln \left[\frac{|z|+\sqrt{R^{2}+z^{2}}}{R}\right]\right) .
\end{aligned}
$$


a)

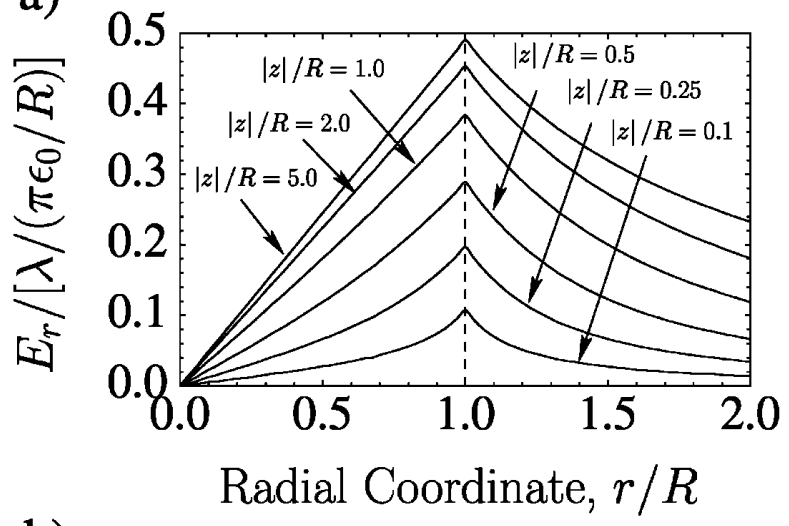

b)

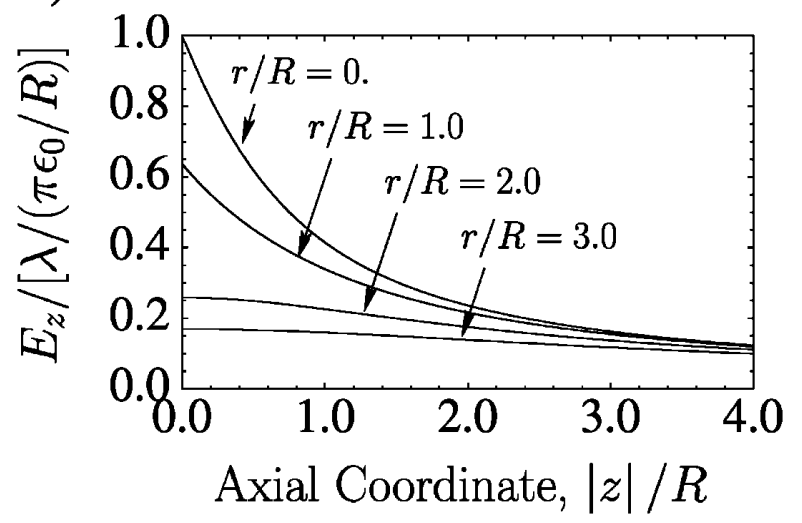

FIG. 3. Radial and axial electric self-field components [Eq. (12)] of a uniform density axisymmetric beam with radius $R=$ const near a conducting plate. In (a) the scaled radial electric field $E_{r} /\left[\lambda /\left(\pi \epsilon_{0} R\right)\right]$ is plotted versus $r / R$ in fixed $z$ planes. In (b) the scaled axial electric field $E_{z} /\left[\lambda /\left(\pi \epsilon_{0} R\right)\right]$ is plotted versus $|z| / R$ in fixed $r$ cylinders.

This divergence is related to the 2D nature of the problem and shows that this model is inadequate for direct use in estimates of axial acceleration induced by the plate. Regularization of this divergence to model image induced self-field accelerations can be carried out by adding a grounded, cylindrical pipe to cut off the self-field interaction range (as would be present in the laboratory) or by using an axially bunched beam model. Even though $\phi$ is diverging in $|z|$ in this simple model, the formula for $E_{r}$ can still be applied in Eqs. (1) and (4) when the beam energy is held fixed because the transverse dynamics do not depend on the absolute scale of $\phi$. Because little fractional change in particle energy will occur in a high-energy beam when the beam is near the plate we will neglect such energy changes in our model induced by $E_{z}$. We analyze only transverse beam effects using the formula for $E_{r}$ and take $\beta_{b}=$ const and $\gamma_{b}=$ const near the plate. A rough estimate for the validity of this approximation can be obtained by requiring that the axial beam energy be much greater than the on-axis potential energy drop experienced by beam particles impinging on the plate from one beam radius in axial distance from the plate. Using Eq. (14) to estimate the potential drop, we obtain

$$
\mathcal{E}_{b} \gg q \phi(r=0, R) \sim \frac{q \lambda}{2 \pi \epsilon_{0} R^{2}}
$$

as a criteria for the validity of neglecting acceleration effects induced by the plate on the transverse envelope.

This simple self-field model can also be used to estimate the scaling in $|z|$ of the transverse potential drop from the radial center $(r=0)$ to the edge $(r=R)$ of the beam. Equation (11) gives

$$
\begin{aligned}
\Delta \phi(z) & \equiv \phi(r=0, z)-\phi(r=R, z) \\
& =\frac{\lambda}{\pi \epsilon_{0}} \int_{0}^{\infty} \frac{d w}{w^{2}}\left(1-e^{-w|z| / R}\right)\left[1-J_{0}(w)\right] J_{1}(w) .
\end{aligned}
$$

Equation (16) provides a reliable estimate for $\Delta \phi$ in physical applications even though $\phi$ diverges in $|z|$ because the potential drop is a relative transverse measure. The potential drop is plotted in Fig. 4. Observe that $\Delta \phi(z)$ rapidly decreases from the limiting value $\lim _{|z| \rightarrow \infty} \Delta \phi=$ $\lambda /\left(4 \pi \epsilon_{0}\right)$ to zero near the plate. This shorting out of the transverse ion beam potential well suggests that any (ideally only small numbers of) electrons trapped in the ion distribution will likely be lost near the diagnostic plane because the electrons are much more mobile than ions and will be radially lost within short axial propagation distances in this region. Biases can also be applied to radial structures far outside of the beam to prevent such electrons from producing cascade interactions back with the beam. Quantifying such effects will become important in diagnostics to measure trapped electron components - a topic not directly addressed in this paper, but of increasing interest in high-intensity beam transport $[13,14]$.

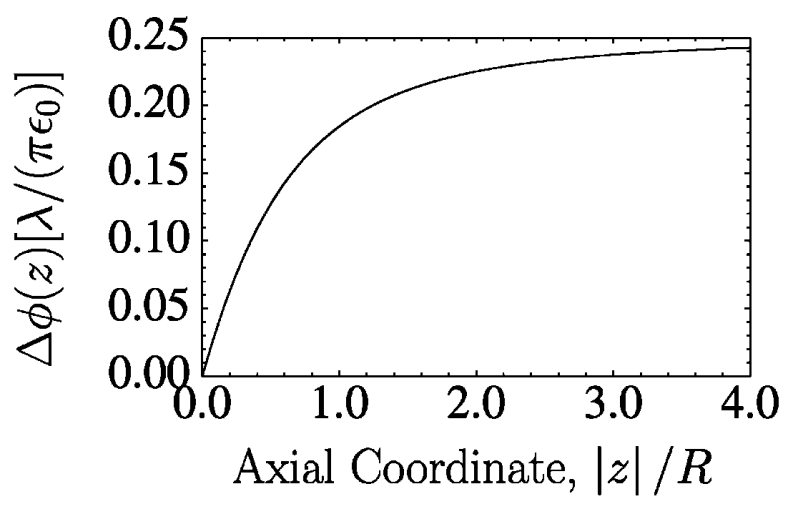

FIG. 4. Radial potential drop of a uniform density axisymmetric beam with radius $R=$ const near a conducting plate. The potential drop $\Delta \phi(z)=\phi(r=0, z)-\phi(r=R, z)$ from beam center $(r=0)$ to edge $(r=R)$ is normalized by $\lambda /\left(\pi \epsilon_{0}\right)$ and plotted versus $|z| / R$. 


\section{B. Corrected envelope equation and results}

We apply the self-field solution above to motivate a simple, corrected envelope equation for an axisymmetric beam with a normally incident centroid impinging on a conducting plate from $z<0$. We take $\kappa_{x}(s)=\kappa_{y}(s) \equiv$ $\kappa(s), \varepsilon_{x}=\varepsilon_{y} \equiv \varepsilon$, and $r_{x}(s)=r_{y}(s) \equiv R(s)$. The form factors (6) are calculated from Eq. (12) as

$$
F_{x}=F_{y}=-\frac{4 \pi \epsilon_{0}}{\lambda}\left\langle r \frac{\partial \phi}{\partial r}\right\rangle_{\perp}=F_{a}\left(\frac{|z|}{R}\right),
$$

where

$$
F_{a}(\zeta) \equiv 8 \int_{0}^{\infty} \frac{d w}{w^{2}}\left(1-e^{-w \zeta}\right) J_{1}(w) J_{2}(w) .
$$

The integral in Eq. (18) can be equivalently calculated as

$$
\begin{aligned}
F_{a}(\zeta) & =2 \zeta^{2}\left[{ }_{2} F_{1}\left(-\frac{1}{2}, \frac{1}{2} ; 2 ;-\frac{4}{\zeta^{2}}\right)-1\right] \\
& =2 \zeta^{2}\left(\frac{2}{\pi} \int_{0}^{1} d t \sqrt{\frac{1-t}{t}} \sqrt{1+\frac{4 t}{\zeta^{2}}}-1\right) .
\end{aligned}
$$

Here, ${ }_{2} F_{1}(a, b ; c ; x)$ is the hypergeometric function with integral representation

$$
{ }_{2} F_{1}(a, b ; c ; x)=\frac{\Gamma(c)}{\Gamma(b) \Gamma(c-b)} \int_{0}^{1} d t t^{b-1}(1-t)^{c-b-1}(1-t x)^{-a},
$$

and $\Gamma(x)=\int_{0}^{\infty} d t t^{x-1} e^{-t}$ is the gamma function. We heuristically apply this form factor to a beam slice with evolving radius $r_{x}(s)=r_{y}(s)=R(s)$ that is at an axial distance $|z|=\left|s-s_{p}\right|$ from the plate to obtain the corrected axisymmetric beam envelope equation

$$
R^{\prime \prime}+\kappa R-\frac{Q}{R} F_{a}\left(\frac{\left|s-s_{p}\right|}{R}\right)-\frac{\varepsilon^{2}}{R^{3}}=0 .
$$

This equation is not self-consistent because the form factor correction is derived for $R=$ const but is applied for evolving $R(s)$. However, the error involved in this approximation is expected to be small unless the envelope radius changes rapidly near the plate.

Rather than directly employing Eqs. (18) or (19) to calculate the nonlinear form factor $F_{a}$, simpler approximate analytical expressions for $F_{a}$ can be calculated as follows. In the beam (i.e., $r \leq R$ and $z \leq 0$ ), the Poisson equation (2) can be expressed as

$$
\left(\frac{1}{r} \frac{\partial}{\partial r} r \frac{\partial}{\partial r}+\frac{\partial^{2}}{\partial z^{2}}\right) \phi(r, z)=-\frac{\lambda}{\pi \epsilon_{0} R^{2}} .
$$

The solution to this equation can be expanded in a power series in $r^{2}$ as

$$
\phi(r, z)=\sum_{\nu=0}^{\infty} f_{2 \nu}(z) r^{2 \nu},
$$

where the $f_{2 \nu}(z)$ are $z$-varying expansion coefficients. Identifying $\phi(r=0, z)=f_{0}(z)$ and requiring that Eq. (21) is satisfied for all powers of $r$ shows that

$$
\begin{aligned}
f_{0}(z) & =\phi(r=0, z) \\
f_{2 \nu+2}(z) & =-\frac{\lambda}{4 \pi \epsilon_{0} R^{2}} \delta_{0, \nu}-\frac{1}{(2 \nu+2)^{2}} \frac{\partial^{2}}{\partial z^{2}} f_{2 \nu}(z),
\end{aligned}
$$

where $\delta_{\mu, \nu}$ is the Kronecker delta function $\left(\delta_{\mu, \nu}=1\right.$ when $\mu=\nu$ and $\delta_{\mu, \nu}=0$ when $\mu \neq \nu$ ). Using the onaxis field $E_{z}(r=0, z)=-\partial \phi(r=0, z) / \partial z$ in Eq. (13) and iterating the recursion between terms in Eq. (23), we obtain a series expansion for $E_{r}(r, z)=-\partial \phi(r, z) / \partial z$ that is valid within the beam:

$$
E_{r}(r, z)=\frac{\lambda}{2 \pi \epsilon_{0} R^{2}}\left\{\frac{|z|}{\sqrt{R^{2}+z^{2}}} r+\sum_{\nu=2,3, \ldots}^{\infty} \frac{(-1)^{\nu} \nu}{2^{2 \nu-2}(\nu !)^{2}} \frac{\partial^{2 \nu-2}}{\partial z^{2 \nu-2}} \frac{z}{\sqrt{R^{2}+z^{2}}} r^{2 \nu-1}\right\} .
$$

The first term of this expansion corresponds to the linear self-field component $\propto r$, and the $\nu=2$ term corresponds to a cubic nonlinear self-field component $\propto r^{3}$. Using the linear and then the linear plus cubic terms of Eq. (24) in Eq. (17) gives

$$
F_{a}(\zeta) \simeq \begin{cases}\frac{\zeta}{\sqrt{1+\zeta^{2}}}, & \text { linear term } \\ \frac{\zeta}{\sqrt{1+\zeta^{2}}}\left[1+\frac{1}{4} \frac{1}{1+\zeta^{2}}\left(1-\frac{\zeta^{2}}{1+\zeta^{2}}\right)\right], & \text { linear plus cubic terms }\end{cases}
$$

The envelope equation (20) with the linear term form factor in Eq. (25) is consistent with taking emittance $\varepsilon=$ const because the self-field is taken to be linear in this approximation.

The full nonlinear [Eq. (18) or Eq. (19)] and approximate [Eq. (25)] form factors are plotted in Fig. 5 versus axial distance from the plate in beam radii $\zeta=|z| / R=$ $\left|s-s_{p}\right| / R$. For large $\zeta$ note that $F_{a} \simeq 1$ and we obtain the usual envelope equations [1], whereas $F_{a}$ rapidly decreases to zero at $\zeta=0$ when $\zeta$ is decreased to values corresponding to axial distances within the order of a beam radius from the plate. This decrease stems from the radial self-field of the beam being shorted out near the conducting plate, resulting in a decrease in the strength of the perveance term in the envelope equation.

A numerical solution to the corrected envelope equation (20) with $\varepsilon=$ const is plotted in Fig. 6 together with the uncorrected solution with $F_{a}=1$. Parameters chosen represent a typical diagnostic measurement in the HCX 


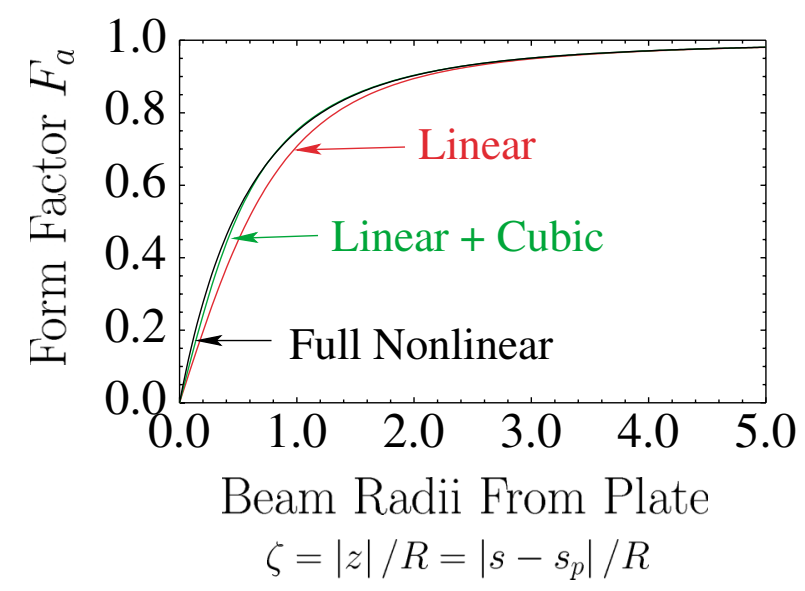

FIG. 5. (Color) Form factor $F_{a}$ for the axisymmetric envelope equation (20) in full nonlinear form [black, Eq. (18) or (19)] and with linear and linear plus cubic nonlinear approximations [red and green, Eq. (25)] plotted versus $\zeta=\left|s-s_{p}\right| / R$.

experiment described in Sec. III and the corrected solution employs the full nonlinear form factor given by Eqs. (18) or (19). In Table I, values of the envelope radius $R$ and angle $R^{\prime}$ at the plate $\left(s=s_{p}\right)$ are contrasted for constant emittance numerical solutions to Eq. (20) for a range of beam parameters and initial conditions. Parameters chosen in the first three groups of rows in Table I include the solution shown in Fig. 6 and represent possible ranges of beam parameters for the HCX experiment and other low-energy quadrupole transport lines for heavy-ion fusion. The last row is a more extreme case representing a possible low-energy solenoidal transport lattice under consideration for heavy-ion fusion applica-
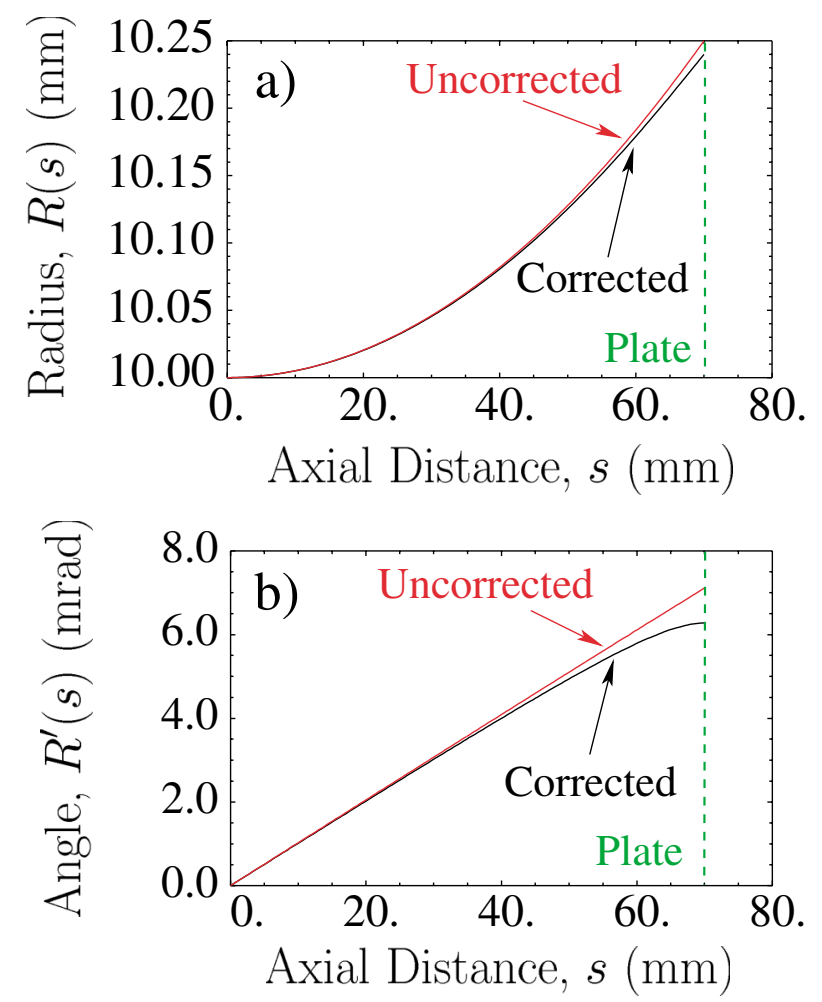

FIG. 6. (Color) Evolution of (a) envelope radius $R$ and (b) angle $R^{\prime}$ given by Eq. (20) for an axisymmetric beam free drift expanding into a conducting plate. Solutions are given for corrected [black, $F_{a}\left(\left|s-s_{p}\right| / R\right)$ ] and uncorrected [red, $\left.F_{a}=1\right]$ evolutions. Beam parameters are $Q=10 \times 10^{-4}$ and $\varepsilon=50 \mathrm{mmmrad}$, the conducting plate is at $s=s_{p}=$ $70 \mathrm{~mm}$, and initial conditions at $s=0$ are $R(0)=10 \mathrm{~mm}$ and $R^{\prime}(0)=0$.

TABLE I. Initial and final envelope radii and angles for an axisymmetric beam free drifting from $s=0$ into a conducting plate at $s=s_{p}=70 \mathrm{~mm}$. Envelope solutions are produced by numerical integration of Eq. (20) with various form factor models for $F_{a}$ and self-consistent 3D PIC simulations are produced with the WARP code. Final envelope radii and angles are tabulated for uncorrected envelope model with $F_{a}=1$, linear corrected envelope model $F_{a}$ [Eq. (25)], full nonlinear envelope model $F_{a}$ [Eq. (18) or (19)], and PIC simulations.

\begin{tabular}{|c|c|c|c|c|c|c|c|c|c|c|c|}
\hline \multicolumn{2}{|c|}{ Beam parameters } & \multicolumn{2}{|c|}{ Initial conditions } & \multicolumn{8}{|c|}{ Final conditions ( $R$ in $\mathrm{mm}, R^{\prime}$ in $\mathrm{mrad}$ ) } \\
\hline \multirow[t]{2}{*}{ Perveance, $Q$} & \multirow{2}{*}{$\begin{array}{c}\text { Emittance, } \varepsilon \\
(\mathrm{mm} \text { mrad })\end{array}$} & \multirow{2}{*}{$\begin{array}{c}R(0) \\
(\mathrm{mm})\end{array}$} & \multirow{2}{*}{$\begin{array}{c}R^{\prime}(0) \\
(\mathrm{mrad})\end{array}$} & \multicolumn{2}{|c|}{$F_{a}=1$} & \multicolumn{2}{|c|}{$F_{a}$ linear } & \multicolumn{2}{|c|}{$F_{a}$ nonlinear } & \multicolumn{2}{|c|}{ 3D PIC } \\
\hline & & & & $R\left(s_{p}\right)$ & $R^{\prime}\left(s_{p}\right)$ & $R\left(s_{p}\right)$ & $R^{\prime}\left(s_{p}\right)$ & $R\left(s_{p}\right)$ & $R^{\prime}\left(s_{p}\right)$ & $R\left(s_{p}\right)$ & $R^{\prime}\left(s_{p}\right)$ \\
\hline $8 \times 10^{-4}$ & 50 & 10 & 0 & 10.20 & 5.73 & 10.19 & 4.99 & 10.19 & 5.07 & 10.2 & 5.0 \\
\hline $8 \times 10^{-4}$ & 50 & 10 & 20 & 11.59 & 25.35 & 11.58 & 24.63 & 11.58 & 24.70 & 11.6 & 24.6 \\
\hline $8 \times 10^{-4}$ & 50 & 10 & 40 & 12.98 & 45.03 & 12.98 & 44.32 & 12.98 & 44.39 & 13.0 & 44.3 \\
\hline $8 \times 10^{-4}$ & 50 & 10 & -20 & 8.81 & -13.80 & 8.80 & -14.56 & 8.80 & -14.49 & 8.8 & -14.5 \\
\hline $8 \times 10^{-4}$ & 50 & 10 & -40 & 7.42 & -33.21 & 7.42 & -34.00 & 7.42 & -33.92 & 7.4 & -33.9 \\
\hline $8 \times 10^{-4}$ & 100 & 10 & 0 & 10.22 & 6.24 & 10.21 & 5.50 & 10.21 & 5.58 & 10.2 & 5.5 \\
\hline $8 \times 10^{-4}$ & 50 & 15 & 0 & 15.13 & 3.77 & 15.12 & 3.06 & 15.12 & 3.13 & 15.1 & 3.1 \\
\hline $5 \times 10^{-4}$ & 50 & 10 & 0 & 10.13 & 3.66 & 10.12 & 3.19 & 10.12 & 3.24 & 10.1 & 3.2 \\
\hline $10 \times 10^{-4}$ & 50 & 10 & 0 & 10.25 & 7.11 & 10.23 & 6.18 & 10.24 & 6.28 & 10.2 & 6.2 \\
\hline $15 \times 10^{-4}$ & 50 & 10 & 0 & 10.37 & 10.54 & 10.36 & 9.16 & 10.36 & 9.30 & 10.3 & 9.2 \\
\hline $100 \times 10^{-4}$ & 125 & 25 & 0 & 25.98 & 27.71 & 25.82 & 19.56 & 25.84 & 20.46 & 25.8 & 19.7 \\
\hline
\end{tabular}


tions [15]. Final values are tabulated for form factors $F_{a}=1 \quad$ (uncorrected), $\quad F_{a}=\left|s-s_{p}\right| / \sqrt{R^{2}+\left(s-s_{p}\right)^{2}}$ [linear field correction, Eq. (25)], and $F_{a}\left(\left|s-s_{p}\right| / R\right)$ [nonlinear correction, Eq. (18) or Eq. (19)]. Negligible differences are observed between envelope solutions produced with the nonlinear form factor and the approximate form factor based on linear plus cubic field terms in Eq. (25). For most applications, deviations between results produced by the linear-field approximation and nonlinear form factors are not significant. The most significant correction for parameters explored is in the envelope angle at the plate $R^{\prime}\left(s_{p}\right)$ with $\sim 1 \mathrm{mrad}$ changes characteristic of perveance ranges possible in HCX-like experiments. In all cases corrections to the envelope coordinate at the plate $R\left(s_{p}\right)$ are very small and not likely resolvable. The values of the final corrected envelope coordinate and angle at the plate depend on the drift length to the plate, the beam emittance $\varepsilon$, and perveance $Q$. These dependencies cannot be scaled away. However, we find that deviations between the corrected and uncorrected envelope angles at the plate increase most strongly with increasing values of $Q$.

Because the envelope angle error induced by the plate is systematic, it can degrade precision beam matching. The angle error in $R^{\prime}$ can be viewed as a mismatch seed that evolves into mismatch excursions in $R$ in subsequent or prior transport. For example, in the continuous focusing approximation [2], it can be shown that a smallamplitude envelope perturbation $\delta R=R-R_{m}$ about a matched beam solution with $R=R_{m}=$ const with finite initial angle error $\delta R^{\prime}(0) \neq 0$ and zero initial coordinate error $\delta R(0)=0$ will lead to maximum envelope perturbation excursions $\max [\delta R]$ expressible in two equivalent forms as

$$
\begin{aligned}
\frac{\max [\delta R]}{R_{m}} & =\frac{\left|\delta R^{\prime}(0)\right|}{\sqrt{Q}} \frac{1}{2\left[1+4 \sigma_{0}^{2} \varepsilon^{2} /\left(Q^{2} L_{p}^{2}\right)\right]^{1 / 4}} \\
& =\frac{\left|\delta R^{\prime}(0)\right|}{\sqrt{Q}} \sqrt{\frac{1-\left(\sigma / \sigma_{0}\right)^{2}}{2+2\left(\sigma / \sigma_{0}\right)^{2}}}
\end{aligned}
$$

Here, in the first form, $\sigma_{0}$ is the phase advance of oscillations of a single particle in the applied focusing over one lattice period $L_{p}$ (in continuous focusing all that matters is the rate of phase accumulation $\sigma_{0} / L_{p}$, but the expression is written in this form to allow extrapolation to periodic focusing lattices). In the second form, $\sigma / \sigma_{0}$ is the ratio of single-particle phase advances in the presence $(\sigma)$ and absence $\left(\sigma_{0}\right)$ of the space charge of a uniformdensity matched beam. The depressed phase advance $\sigma$ is calculated from the matched beam in the periodic lattice. The space-charge depression $\sigma / \sigma_{0}$ is a function of $\left[\sigma_{0} \varepsilon /\left(Q L_{p}\right)\right]^{2}$ and satisfies $\lim _{Q \rightarrow 0} \sigma / \sigma_{0}=1$ at zero beam intensity and $\sigma / \sigma_{0}=0$ at the space-charge limit. Results in Ref. [2] establish that Eq. (26) is accurate for the breathing envelope mode in periodic focusing chan- nels with $\sigma_{0}<90^{\circ}$. Stability requirements dictate that this restriction in $\sigma_{0}$ will be well satisfied for most intense beam transport channels [16]. Equation (26) is consistent with $\delta R^{\prime} \sim 1 \mathrm{mrad}$ errors leading to a maximum ( $\sigma=0$, maximum beam intensity) fractional mismatch amplitude of $\max [\delta R] / R_{m} \sim 2.5 \%$ for $Q=$ $8 \times 10^{-4}$.

Self-consistent WARP PIC simulations were also carried out for beam envelope model solutions presented in Table I and results are also summarized in Table I. General features of the simulations are presented in Sec. III. Simulation parameters in addition to the varied beam parameters listed in the table are given there. The envelope coordinates and angles presented are statistically calculated from the simulated particle distribution with $R=2^{1 / 2}\left\langle x^{2}+y^{2}\right\rangle_{\perp}^{1 / 2}$ and $R^{\prime}=2^{1 / 2}\left\langle x x^{\prime}+y y^{\prime}\right\rangle_{\perp} /\left\langle x^{2}+\right.$ $\left.y^{2}\right\rangle_{\perp}^{1 / 2}$. Potential contours of a simulation are shown in Fig. 7. The contours clearly show the strong influence of the plate on the beam self-field. The simulations agree well with the envelope model results for the small angle corrections and provide strong support for the accuracy of the reduced envelope models derived. Indeed, the level of agreement is surprising for the cases with larger initial envelope angles $R^{\prime}(0)$ because the envelope model form factors are derived taking $R=$ const and therefore do not consistently take into account changes in the envelope radius near the plate. The transverse beam emittances $\varepsilon_{x}$

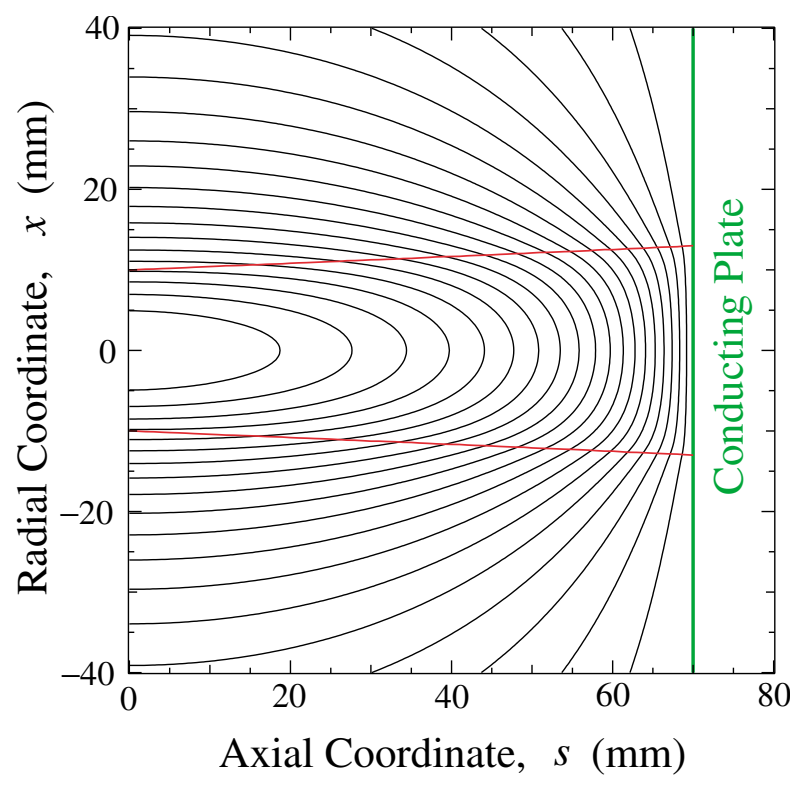

FIG. 7. (Color) Self-field potential contours of $\phi$ and statistical beam envelope $R$ of a WARP PIC simulation of a midpulse axisymmetric beam near a conducting plate. Contours in $\phi$ are in the $x-z$ plane $(y=0)$ and are equally spaced in volts, and the statistical envelope projections $x= \pm R= \pm 2\left\langle x^{2}\right\rangle_{\perp}^{1 / 2}$ are shown in red. Beam parameters correspond to those in Table I with $Q=8 \times 10^{-4}, \varepsilon=50 \mathrm{~mm} \mathrm{mrad}, R(0)=10 \mathrm{~mm}$, and $R^{\prime}(0)=40 \mathrm{mrad}$. 
and $\varepsilon_{y}$ were statistically calculated from the simulated particle distribution using Eq. (7) and typically had $\sim 1 \%-2 \%$ variations (both increases and decreases) along the axial length of the simulations. These variations were dominated by statistical noise and other numerical errors. The magnitudes of the emittance variations related to nonlinear self-fields of the beam are both too small and too near the plate to induce significant changes in the beam envelope. Little change in simulation results is obtained when the pipe radius of the grounded cylindrical pipe is increased or decreased by factors of 2 and more. Finally, it is interesting to note from Table I that corrected envelope model results derived with linear self-field form factor corrections for $F_{a}$ [Eq. (25)] agree better with the simulations than envelope model results obtained with the form factor $F_{a}$ derived from the full nonlinear selffield model [Eqs. (17) and (19)]. This provides an explicit illustration of the general point made in Sec. II that inconsistent nonlinear corrections to the form factors $F_{x}$ and $F_{y}$ without the correct emittance evolutions in the nonlinear fields need not result in improved modeling.

\section{CORRECTED ENVELOPE EQUATIONS FOR ELLIPTICAL BEAMS}

Calculation of the form factors in Eq. (6) to obtain corrected envelope equations for beams of elliptical cross section $\left(r_{x} \neq r_{y}\right)$ is considerably more complicated than for the axisymmetric beams analyzed in Sec. IV. However, using the axisymmetric beam results as a guide to motivate model approximations, we develop a simple model that recovers most of the effect of the plates on elliptical beams.

\section{A. Self-field solution}

To model the beam self-fields, we assume a uniformdensity, normally incident beam of elliptical cross section with edge radii $r_{x}=$ const and $r_{y}=$ const along the $x$ and $y$ axes. In this case the beam density is

$$
n(x, y, z)=\frac{\lambda}{\pi q r_{x} r_{y}} \Theta\left(1-\frac{x^{2}}{r_{x}^{2}}-\frac{y^{2}}{r_{y}^{2}}\right) \Theta(-z)
$$

The 3D Poisson equation (2) is approximated within the beam (i.e., $x^{2} / r_{x}^{2}+y^{2} / r_{y}^{2} \leq 1$ and $z \leq 0$ ) as

$$
\begin{aligned}
\left(\frac{\partial^{2}}{\partial x^{2}}+\frac{\partial^{2}}{\partial y^{2}}\right) \phi & \simeq-\frac{\lambda}{\pi r_{x} r_{y} \epsilon_{0}}+\frac{\partial E_{z}}{\partial z}(r=0, z) \\
& \equiv-\frac{\lambda_{e}}{\pi r_{x} r_{y} \epsilon_{0}},
\end{aligned}
$$

where we calculate the on-axis electric field $E_{z}(r=$ $0, z)=-\partial \phi(r=0, z) / \partial z$ exactly from Eqs. (9) and (27) and obtain

$$
\begin{aligned}
E_{z}(r=0, z)=\frac{\lambda}{\pi \epsilon_{0}}( & \int_{-\pi}^{\pi} \frac{d \theta}{2 \pi} \frac{\sqrt{r_{x}^{2} \cos ^{2} \theta+r_{y}^{2} \sin ^{2} \theta+z^{2}}}{r_{x}^{2} \cos ^{2} \theta+r_{y}^{2} \sin ^{2} \theta} \\
& \left.-\frac{|z|}{r_{x} r_{y}}\right) .
\end{aligned}
$$

Equation (29) is derived by differentiating Eq. (9) with respect to $z$, evaluating the result at $r=0$, and then taking $\tilde{x}=r_{x} \tilde{\rho} \cos \tilde{\theta}, \quad \tilde{y}=r_{y} \tilde{\rho} \sin \tilde{\theta}$, and $d^{3} \tilde{x}=$ $r_{x} r_{y} d \tilde{\rho} \tilde{\rho} d \tilde{\theta} d \tilde{z}$ and carrying out integrals with respect to $\tilde{z}$ and $\tilde{\rho}$. As a partial check of Eq. (29), observe that for a round beam with $r_{x}=r_{y}=R$ that this expression reduces to the on-axis field of the axisymmetric beam previously calculated in Eq. (13). Using Eq. (29), we calculate the corrected line-charge density $\lambda_{e}$ in Eq. (28) in several equivalent forms as

$$
\begin{aligned}
\lambda_{e} & \equiv \lambda-\pi r_{x} r_{y} \epsilon_{0} \frac{\partial E_{z}}{\partial z}(r=0, z) \\
& =\frac{2 \lambda}{\pi} \frac{r_{y}|z|}{r_{x} \sqrt{r_{x}^{2}+z^{2}}} \Pi\left(\frac{r_{x}^{2}-r_{y}^{2}}{r_{x}^{2}} \mid \frac{r_{x}^{2}-r_{y}^{2}}{r_{x}^{2}+z^{2}}\right) \\
& =\frac{2 \lambda}{\pi} \frac{r_{x}|z|}{r_{y} \sqrt{r_{y}^{2}+z^{2}}} \Pi\left(-\frac{r_{x}^{2}-r_{y}^{2}}{r_{y}^{2}} \mid-\frac{r_{x}^{2}-r_{y}^{2}}{r_{y}^{2}+z^{2}}\right) .
\end{aligned}
$$

Here, $\Pi(a \mid b)$ is the complete elliptic integral of the third kind defined by $\Pi(a \mid b) \equiv \int_{0}^{\pi / 2} d \theta(1-$ $\left.a \sin ^{2} \theta\right)^{-1}\left(1-b \sin ^{2} \theta\right)^{-1 / 2}$.

Because the corrected density $\lambda_{e} /\left(\pi r_{x} r_{y}\right)$ in Eq. (30) is independent of $x$ and $y$, the solution to Eq. (28) consistent with a regular external solution at large radius $r$ can be obtained by rescaling the usual transverse 2D field solution of a uniform-density elliptical beam [2,17] with density $\lambda /\left(\pi r_{x} r_{y}\right)$ by replacing $\lambda \rightarrow \lambda_{e}$ in the usual expressions. This gives within the beam

$$
\begin{aligned}
& -\frac{\partial \phi}{\partial x}=\frac{\lambda_{e}}{\pi \epsilon_{0}} \frac{1}{r_{x}+r_{y}} \frac{x}{r_{x}}, \\
& -\frac{\partial \phi}{\partial y}=\frac{\lambda_{e}}{\pi \epsilon_{0}} \frac{1}{r_{x}+r_{y}} \frac{y}{r_{y}} .
\end{aligned}
$$

\section{B. Corrected envelope equations and results}

Using the approximate field solutions in Eq. (31), we calculate the form factors in Eq. (6) for the elliptical beam as

$$
F_{x}=F_{y} \equiv F_{e}=\frac{\lambda_{e}}{\lambda} .
$$

Then using Eq. (30), $F_{e}$ can be expressed in symmeterized form as

$$
F_{e}(\zeta, \epsilon)=\frac{2}{\pi} \frac{\zeta}{\epsilon \sqrt{\epsilon+\zeta^{2}}} \Pi\left(1-\frac{1}{\epsilon^{2}} \mid \frac{\epsilon-1 / \epsilon}{\epsilon+\zeta^{2}}\right) .
$$

Here, $\epsilon \equiv r_{x} / r_{y}$ is the ellipticity of the envelope and $\zeta \equiv$ $|z| / \sqrt{r_{x} r_{y}}$ is the axial distance to the plate in average 


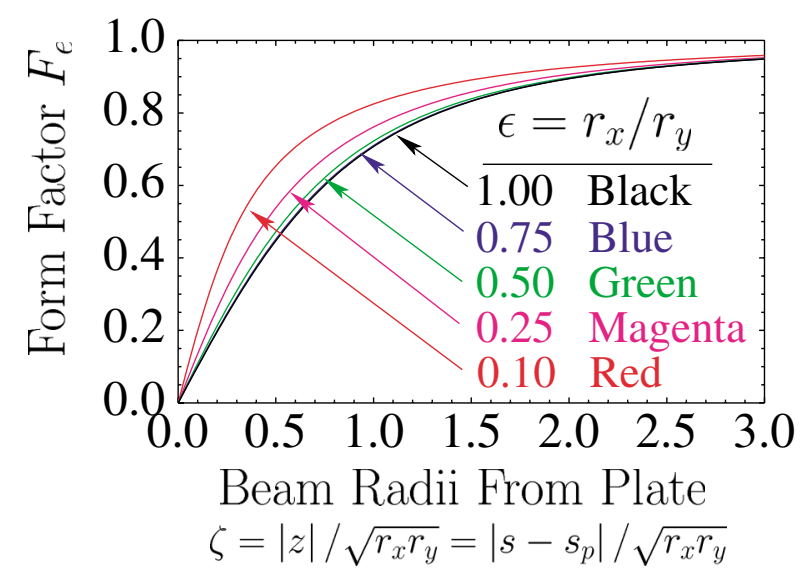

FIG. 8. (Color) Form factor $F_{e}$ [Eq. (33)] for the elliptical beam envelope equations (34) plotted versus $\zeta=|z| / \sqrt{r_{x} r_{y}}=$ $\left|s-s_{p}\right| / \sqrt{r_{x} r_{y}}$ for values of $\epsilon=r_{x} / r_{y}$ indicated.

transverse beam radii. These form factors can be checked in several limits. First, using $\Pi(a \mid 0)=\pi /(2 \sqrt{1-a})$, $\lim _{|z| \rightarrow \infty} F_{e}=1$ follows, and far from the plate the usual form factors for a $2 \mathrm{D}$ elliptical beam in free space are obtained. Next, using $\Pi(0 \mid 0)=\pi / 2$, in the limit of a round beam with $r_{x}=r_{y}=R, F_{e}=|z| / \sqrt{R^{2}+z^{2}}$ consistent with the linear-field axisymmetric beam result given in Eq. (25) with $\zeta=|z| / R$. The form factor $F_{e}(\zeta, \epsilon)$ is plotted in Fig. 8 versus $\zeta=$ $|z| / \sqrt{r_{\mathrm{x}</ \mathrm{m}: \mathrm{mi}>} r_{y}}=\left|s-s_{p}\right| / \sqrt{r_{x} r_{y}}$ for values of $\epsilon=$ $r_{x} / r_{y}$. Because $F_{e}$ is invariant under the replacement $\epsilon \rightarrow$ $1 / \epsilon$, only values of $\epsilon \leq 1$ are shown. Qualitatively, the results are similar to the axisymmetric beam results presented in Sec. IV B and there is little variation of $F_{e}$ in $\epsilon$ for all but the most extreme values of ellipticity $\epsilon=$ $r_{x} / r_{y} \neq 1$
To obtain corrected envelope equations for an elliptical beam near a conducting plate, analogously to the axisymmetric case in Sec. IV, in Eq. (4) we heuristically apply the form factors (33) with $|z|=\left|s-s_{p}\right|$ the distance from the conducting plate and evolving beam radii $r_{x}$ and $r_{y}$ giving

$$
\begin{aligned}
& r_{x}^{\prime \prime}+\kappa_{x} r_{x}-\frac{2 Q}{r_{x}+r_{y}} F_{e}\left(\frac{\left|s-s_{p}\right|}{\sqrt{r_{x} r_{y}}}, \frac{r_{x}}{r_{y}}\right)-\frac{\varepsilon_{x}^{2}}{r_{x}^{3}}=0, \\
& r_{y}^{\prime \prime}+\kappa_{y} r_{y}-\frac{2 Q}{r_{x}+r_{y}} F_{e}\left(\frac{\left|s-s_{p}\right|}{\sqrt{r_{x} r_{y}}}, \frac{r_{x}}{r_{y}}\right)-\frac{\varepsilon_{y}^{2}}{r_{y}^{3}}=0 .
\end{aligned}
$$

Because of the weak dependence of $F_{a}(\zeta, \epsilon)$ on $\epsilon=r_{x} / r_{y}$, in many cases adequate precision can be attained by approximating $F_{e}$ in Eq. (34) as

$$
F_{e}(\zeta, \epsilon) \simeq F_{e}(\zeta, 1)=\frac{\zeta}{\sqrt{1+\zeta^{2}}}
$$

with $\zeta=\left|s-s_{p}\right| / \sqrt{r_{x} r_{y}}$. Note that $F_{e}(\zeta, 1)$ is the same form factor as for the axisymmetric beam in the linear self-field approximation [see Eq. (25)] with $\zeta=|z| / R$ for the axisymmetric beam replaced by $\zeta=|z| / \sqrt{r_{x} r_{y}}$ for the elliptical beam.

Results of the corrected envelope model using Eq. (33) for $F_{e}$ in numerical integrations of Eq. (34) with $\varepsilon_{x}=$ $\varepsilon_{y}=$ const are contrasted to uncorrected envelope model results with $F_{e}=1$ and self-consistent 3D WARP PIC simulations in Table II. Geometry and beam parameters are analogous to those presented in Table I. Results are grouped for two separate values of perveance $Q$ showing three initial conditions for each value. The first row in each group is an axisymmetric initial condition directly comparable to cases in Table I for consistency checks. In

TABLE II. Initial and final envelope radii and angles for axisymmetric and elliptical beam initial conditions free drifting from $s=0$ into a conducting plate at $s=s_{p}=70 \mathrm{~mm}$. Final envelope radii are shown for the following: uncorrected envelope with $F_{e}=1$, linear corrected $F_{e}$, and 3D self-consistent WARP PIC simulations. Upper and lower table entries have different values of perveance $Q$.

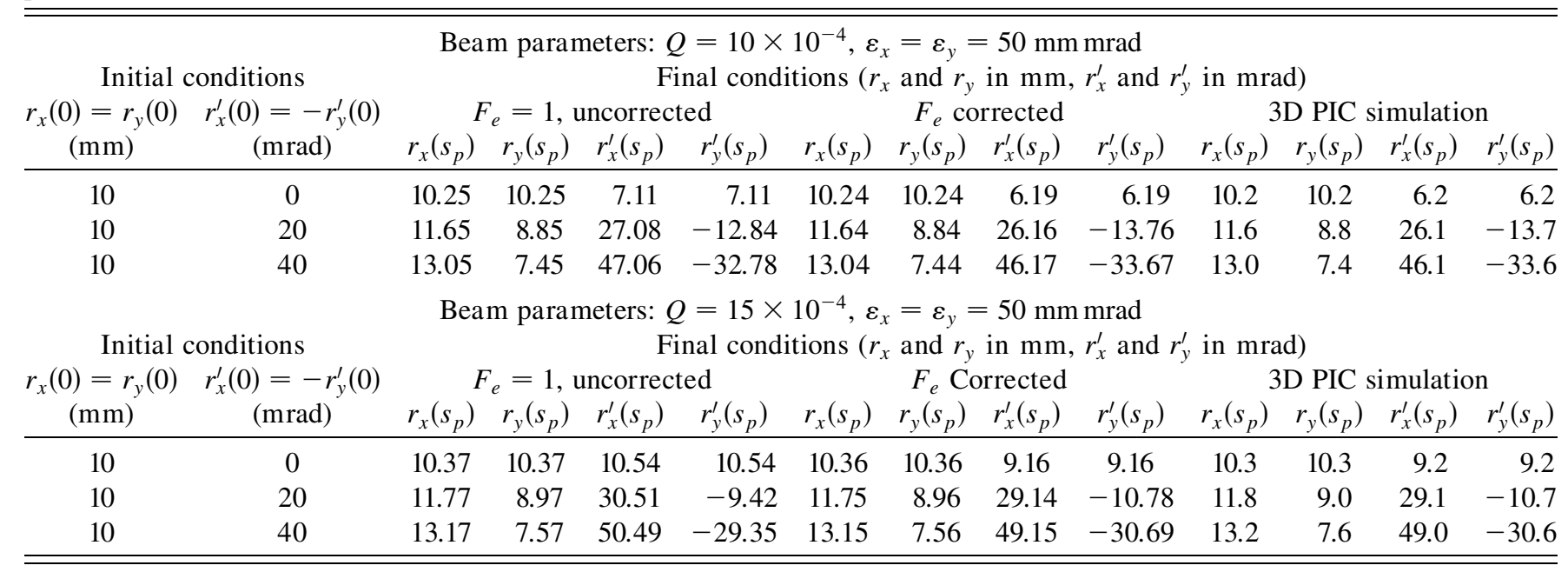


TABLE III. Maximum envelope mismatch excursions seeded from errors associated with intercepting beam diagnostics in a periodic alternating gradient transport lattice describing the HCX experiment. Results are for $\varepsilon_{x}=\varepsilon_{y}=50 \mathrm{~mm} \mathrm{mrad,} \sigma_{0}=80^{\circ}$, and a $70 \mathrm{~mm}$ axial drift from the plate to a midquadrupole drift location.

\begin{tabular}{ccccc}
\hline \hline \multicolumn{2}{c}{ Beam parameters } & \multicolumn{2}{c}{ Mid-drift errors (mrad) } & \multicolumn{2}{c}{ Mismatch Amplitude } \\
$Q$ & $\sigma / \sigma_{0}$ & $\delta r_{x}^{\prime}$ & $\delta r_{y}^{\prime}$ & $\max \left[\delta r_{x} / r_{x m}, \delta r_{y} / r_{y m}\right]$ \\
\hline $1.0 \times 10^{-4}$ & 0.733 & 0.10 & 0.10 & 0.005 \\
$2.5 \times 10^{-4}$ & 0.483 & 0.24 & 0.24 & 0.012 \\
$5.0 \times 10^{-4}$ & 0.288 & 0.47 & 0.47 & 0.020 \\
$7.5 \times 10^{-4}$ & 0.201 & 0.70 & 0.70 & 0.025 \\
$10.0 \times 10^{-4}$ & 0.153 & 0.92 & 0.92 & 0.030 \\
$15.0 \times 10^{-4}$ & 0.078 & 1.35 & 1.35 & 0.037 \\
$20.0 \times 10^{-4}$ & 0.078 & 1.78 & 1.78 & 0.042 \\
\hline \hline
\end{tabular}

the simulations, the envelope radii and angles are statistically calculated from the particle distribution as $r_{x}=$ $2\left\langle x^{2}\right\rangle_{\perp}^{1 / 2}, \quad r_{y}=2\left\langle y^{2}\right\rangle_{\perp}^{1 / 2}$ and $r_{x}^{\prime}=2\left\langle x x^{\prime}\right\rangle_{\perp} /\left\langle x^{2}\right\rangle_{\perp}^{1 / 2}, \quad r_{y}^{\prime}=$ $2\left\langle y y^{\prime}\right\rangle_{\perp} /\left\langle y^{2}\right\rangle_{\perp}^{1 / 2}$. Note for the axisymmetric initial conditions that the $F_{e}=1$ and $F_{e}$ corrected envelope solutions in Table II are identical to the $F_{a}=1$ and $F_{a}$ linear envelope solutions in Table I. The good agreement on the final envelope angles between the simulation results and corrected envelope model results in Table II verifies that the approximate self-field form factor correction in Eq. (33) is adequate for most purposes.

To better understand the impact of these corrections on precision matching of a beam with high space-charge intensity in an alternating gradient quadrupole transport lattice, we analyze the HCX experiment as a practical example. The symmetric doublet (FODO) lattice of the HCX is described in detail in Ref. [2] and here we model the lattice in a simple hard-edge approximation with lattice period $L_{p}=435.2 \mathrm{~mm}$ and quadrupole axial length $0.6949 L_{p} / 2$. Focusing strengths are adjusted for an undepressed particle phase advance of $\sigma_{0}=80^{\circ}$ per period-a typical operating point for a $\mathcal{E}_{b}=1 \mathrm{MeV} K^{+}$ beam. We assume that a slit diagnostic is located $70 \mathrm{~mm}$ downstream of the midaxial drift between two quadrupoles in the periodic lattice with the downstream quadrupoles removed. This is a typical value employed and the drifts range from $60-120 \mathrm{~mm}$ in the experiment depending on the specific diagnostic apparatus employed and the location in the machine lattice. The periodic matched beam envelope $r_{x}=r_{x m}, r_{y}=r_{y m}$ with

$$
r_{x m}\left(s+L_{p}\right)=r_{x m}(s), \quad r_{y m}\left(s+L_{p}\right)=r_{y m}(s),
$$

is calculated in the periodic lattice in the absence of intercepting diagnostics. Matched beam envelope coordinates $\left(r_{x}, r_{x}^{\prime}, r_{y}, r_{y}^{\prime}\right)$ are advanced from the midquadrupole drift to the plate without $\left(F_{e}=1\right)$ plate corrections to the perveance to correspond to the uncorrected diagnostic measurement one would anticipate to obtain for a matched beam envelope. These envelope coordinates are then integrated back to the mid-drift symmetry point using the plate corrected envelope equations $\left(F_{e} \equiv \zeta / \sqrt{1+\zeta^{2}}\right)$ approximating the physical situation where the diagnostic influences the beam evolution. Finally, these envelope coordinates with plate induced mismatch are integrated forward from a midquadrupole location in the periodic HCX lattice using the uncorrected envelope equations $\left(F_{e}=1\right)$ to calculate the envelope mismatch evolution [2]

$$
\delta r_{x}=r_{x}-r_{x m}, \quad \delta r_{y}=r_{y}-r_{y m} .
$$

The advance is carried out at a sufficient number of lattice periods to calculate the maximum envelope mismatch excursions in $\left|\delta r_{x}\right| / r_{x m}$ and $\left|\delta r_{y}\right| / r_{y m}$. Results are summarized in Table III for a range of perveance values. Included in the table are values of $\sigma / \sigma_{0}$ for the periodic matched beam ( $\sigma$ is the particle phase advance in the lattice in the presence of uniform beam space charge) to better illustrate relative space-charge intensity. The induced mismatch angles at the midquadrupole drift are also tabulated. Mismatch errors in the envelope coordinate are negligible in all cases at the mid-drift point but evolve due to the induced angle errors. For the HCX, $Q=$ $7-10 \times 10^{-4}$, and the uncorrected diagnostic effect can induce up to $3 \%$ mismatch amplitudes. Uncorrected measurements introduce systematic envelope errors at all intercepting diagnostics - both where the beam is measured for use in adjusting focusing to improve the match and where the corrected envelope is measured. Uncorrected errors at both locations can increase the ultimately attained mismatch amplitude. Increasing the drift distance to the diagnostic also increases the mismatch because the beam becomes more space-charge dominated (space-charge forces dominate thermal forces) as it drift expands in the absence of applied focusing forces. Mismatches in Table III are somewhat larger than what one would infer from the angle errors and the continuous focusing estimate based on Eq. (26). This deviation results from alternating gradient effects not being properly modeled at higher values of $\sigma_{0}$ and from the fact that the mismatch can project on both breathing 
and quadrupole envelope modes in the alternating gradient lattice while the formula assumes a pure breathing mode projection [2]. Results are not compared to HCX experimental data because the present perveance in the experiment of $Q \simeq 8 \times 10^{-4}$ results in envelope angle corrections induced by the plate that are only near diagnostic thresholds of $\sim 1 \mathrm{mrad}[6,8]$. Therefore meaningful comparisons between theory and experimental data require careful evaluation of error sources due to pulse variations, excitation of focusing optics, electron effects, and other nonideal effects. This is beyond the scope of this theoretical study where we seek to quantify the magnitude and characteristics of the effect under idealized conditions.

\section{CONCLUSIONS}

Generalized transverse envelope equations were derived to improve modeling of ion beams with high space-charge intensity impinging at normal incidence on a conducting plate. Such intercepting plates are typical in intense beam diagnostics used to measure the transverse phase space of the particle distribution of the beam. The corrected envelope equations were derived for both beams of axisymmetric and elliptical transverse cross section by deriving analytical form factor corrections to the perveance term of the usual envelope equations. Predictions of this envelope model were verified using self-consistent 3D PIC simulations. It was found that form factors derived under the approximation of simple linear models of the beam self-field had adequate accuracy for most applications. For usual parameters, the main effect of the plate is a small, systematic correction in the envelope angle at the plate. This effect is a strong function of the beam perveance. Taking account of this effect enables modest improvements in precision beam matching for parameters typical of present experiments and promises more significant matching improvements for future applications with higher perveance.

\section{ACKNOWLEDGMENTS}

The authors wish to thank P. A. Seidl and L. Prost for motivating the need for this study and providing input on examples derived from the HCX experiment, E. P. Lee for suggesting methods that led to several approximations developed, D. P. Grote and J.-L. Vay for help with the WARP PIC simulations, and J. J. Barnard and A. Friedman for useful discussions. This research was performed under the auspices of the U.S. Department of Energy by the University of California at the Lawrence Livermore and Lawrence Berkeley National Laboratories under
Contracts No. W-7405-Eng-48 and No. DE-AC0376SF00098.

[1] M. Reiser, Theory and Design of Charged Particle Beams (John Wiley \& Sons, Inc., New York, 1994).

[2] S. M. Lund and B. Bukh, Phys. Rev. ST Accel. Beams 7, 024801 (2004).

[3] F. J. Sacherer, IEEE Trans. Nucl. Sci. 18, 1105 (1971).

[4] I. Kapchinskij and V. Vladimirskij, in Proceedings of the International Conference on High Energy Accelerators and Instrumentation (CERN Scientific Information Service, Geneva, 1959), p. 274.

[5] V. P. Karpenko, P. A. Seidl, R. M. Franks, and S. M. Lund, in Proceedings of the 2001 Particle Accelerator Conference, Chicago, IL (IEEE, Piscataway, NJ, 2001), p. 1447.

[6] P. A. Seidl, D. Baca, F. M. Bieniosek, A. Faltens, S. M. Lund, A.W. Molvik, L. R. Prost, D. B. Shuman, and W. L. Waldron, Laser Part. Beams 20, 435 (2002).

[7] F. Bieniosek, W. B. Ghiorso, and L. Prost, in Proceedings of the 2003 Particle Accelerator Conference, Portland, OR (IEEE, Piscataway, NJ, 2003), p. WPPB050.

[8] P. A. Seidl et al., in Proceedings of the 2003 Particle Accelerator Conference, Portland, OR (Ref. [7]), p. ROAC001.

[9] D. P. Grote, A. Friedman, I. Haber, W. Fawley, and J.-L. Vay, Nucl. Instrum. Methods Phys. Res., Sect. A 415, 428 (1998).

[10] S. M. Lund, J. J. Barnard, and E. P. Lee, in Proceedings of the XX International Linac Conference, Monterey, CA, 2000 (Stanford Linear Accelerator Center, Stanford, CA, 2001), pp. 290 and MOE11.

[11] S. M. Lund and B. Bukh, in Proceedings of the 2003 Particle Accelerator Conference, Portland, OR (Ref. [7]), p. WPPG022.

[12] J. D. Jackson, Classical Electrodynamics (John Wiley \& Sons, Inc., New York, 1975), 2nd ed.

[13] A.W. Molvik et al., in Proceedings of the 2003 Particle Accelerator Conference, Portland, OR (Ref. [7]), p. TOPC004.

[14] R. H. Cohen, S. M. Lund, A.W. Molvik, M. A. Furman, J.-L. Vay, and P. Stoltz, in Proceedings of the 2003 Particle Accelerator Conference, Portland, OR (Ref. [7]), p. TOAA010.

[15] E. Lee and R. J. Briggs, Lawrence Berkeley National Laboratory Technical Report No. LBNL-40774, UC419, 1997.

[16] M. G. Tiefenback, Ph.D. thesis, University of California at Berkeley, 1986.

[17] R. C. Davidson and H. Qin, Physics of Intense Charged Particle Beams in High Energy Accelerators (World Scientific, New York, 2001). 